\section{Variability in Visual Assessment of Cerebral Aneurysms Could Be Reduced by Quantification of Recurrence Volumes}

After endovascular coil embolization of an intracranial aneurysm, coil compaction, aneurysm growth, or migration of the coil into intraluminal thrombus might lead to recurrence. In this case, interventional neuroradiologists have to decide whether to treat the recurrent aneurysm. Although there are several factors influencing the individual treatment decision, such as aneurysm shape, initial aneurysm size, dynamic aneurysm growth, patient preference, individual treatment risk, patient age, and so forth, previous observations suggest that mainly the recurrence volume determines the treatment decision. ${ }^{1}$

We have read the article of Daugherty et al entitled "Observer Agreement Regarding the Necessity of Retreatment of Previously Coiled Recurrent Cerebral Aneurysms" ${ }^{2}$ with special interest. The authors evaluated observer agreement with respect to the question of whether to treat recurrent aneurysms. The experienced interventionalists (some were even from the same "school") revealed substantial variability in their opinions about the indication for treatment of recurrent aneurysms.

First, this observation reflects the notorious difficulties of clinical therapy decision-making in these conditions. A certain morphology or size suggesting definite need for retreatment is unclear because hemorrhage from residual or recurrent aneurysms is a rare event. Second, the authors put the use of retreatment as an objective end point in clinical trails into question on the basis of the considerable disagreement. Therefore, it would be desirable to define reliable parameters that help to decide whether retreatment is indicated and to consequently assess objective end points in clinical trails (eg, for the evaluation of new coils). We believe that the general use of 2D digital subtraction angiography images for the evaluation of the genuine 3D structure "recurrent aneurysm appearance" is one major source of heterogeneity. The personal calibration of visual preconception by a specific "school" could be another explanation.

In our opinion, evaluation by visual inspection as used in core labs could be replaced by quantitative volumetric parameters. Recently, such a method for the extraction of a quantitative parameter characterizing recurrent aneurysms was proposed and validated. ${ }^{3}$ Briefly, the aneurysm-recurrence volume is estimated by rigid coregistration of two 3D time-of-flight MR angiography (TOF-MRA) datasets acquired at 2 discrete time points after therapy. For this estimation, the vascular structures are automatically segmented ${ }^{4}$ in both datasets and used for a masked intensity-based registration. After registration, the aligned vessel segmentations are subtracted such that the aneurysmrecurrence volume is left over, which can be used for quantification purposes (Fig 1).
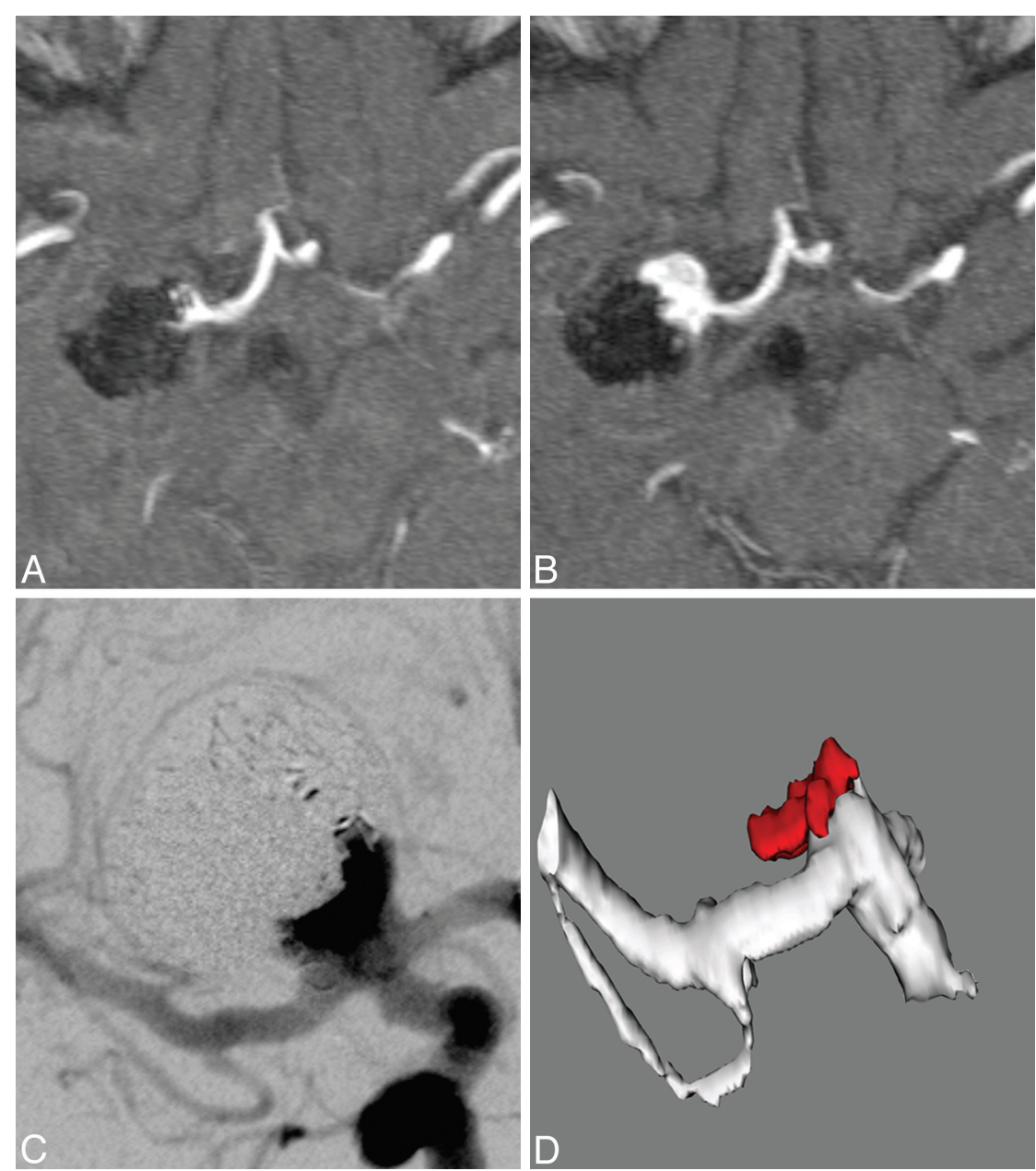

Fig 1. Recurrence of a carotid terminus aneurysm after endovascular coil treatment. Baseline TOF-MRA after endovascular coiling $(A)$ and at 1-year follow-up $(B)$ show recurrence. Conventional cerebral angiogram $(C)$ and computer-aided $3 D$ aneurysm-recurrence volume quantification based on TOF-MRA image sequences (D). The difference between the baseline and follow-up dataset indicates a coil condensation and consecutive reperfusion of the aneurysm (red). 
TOF-MRA is a standard follow-up method after endovascular aneurysm therapy in many centers worldwide. The key difficulties in the application of 3D MRA image sequences for aneurysm-recurrence volume quantification have been the statistical basis of possible outcome variables as well as the development of an algorithm that allows the precise coregistration of routine MRA scans in $3 \mathrm{D}$ space. Such a method needs to be both robust and precise, even if the image data are from different MR imaging scanners.

When applied correctly, such volume measurements can be used as reproducible end points in clinical trails. However, to this point, these measurements are not suitable for clinical decision-making.

A volumetric surrogate end point could substantially decrease sample size by limiting the variability in the assessment as described by Daugherty et al. ${ }^{2}$ Such analysis might allow a more timely estimation of possible benefits of new materials at a fraction of the cost of a study with hundreds of patients that is normally required, at least in part, to reduce the variability introduced by visual assessment.

\section{References}

1. Ries $\mathrm{T}, \mathrm{W}$ ulff $\mathrm{A}, \mathrm{Wegscheider} \mathrm{K}$, et al. Unexpected kink in aneurysm recurrence volume distribution is associated with retreatment decision. Neuroradiology 2010;52:657-58. Epub 2010 Apr 22

2. Daugherty WP, Rad AE, White JB, et al. Observer agreement regarding the necessity of retreatment of previously coiled recurrent cerebral aneurysms. AJNR Am J Neuroradiol 2011;32:566-69

3. Ries T, Wegscheider K, Wulff A, et al. Quantification of recurrence volumes after endovascular treatment of cerebral aneurysm as surrogate endpoint for treatment stability. Neuroradiology 2011;53:593-98

4. Forkert ND, Schmidt-Richberg A, Fiehler J, et al. Fuzzy-based vascular structure enhancement in time-of-flight MRA images for improved segmentation. Methods Inf Med 2011;50:74-83

M. Groth

J. Fiehler

Department of Diagnostic and Interventional Neuroradiology

N.D. Forkert

Department of Medical Informatics University Medical Center Hamburg-Eppendorf

Hamburg, Germany 